lower albumin at presentation predicted critical care admission and mortality. Non-white ethnicity predicted critical care admission but not death. Social deprivation was not predictive of outcome. A risk score was developed incorporating twelve characteristics: age $>40$, male, non-white ethnicity, oxygen saturations $<93 \%$, radiological severity score $>3$, neutrophil count $>8.0 \times 10^{9} / \mathrm{L}$, CRP $>40 \mathrm{mg} / \mathrm{L}$, albumin $<34 \mathrm{~g} / \mathrm{L}$, creatinine $>100 \mu \mathrm{mol} / \mathrm{L}$, diabetes mellitus, hypertension and chronic lung disease. Risk scores of 4 or higher corresponded to a 28-day cumulative incidence of critical care admission or death of $40.7 \%$ (95\% CI: 37.1 to 44.4 ), versus 12.4\% (95\% CI: 8.2 to 16.7$)$ for scores less than 4.

Conclusion Our study identified predictors of critical care admission and death in people admitted to hospital with COVID-19. These predictors were incorporated into a risk score that will inform clinical care and stratify patients for clinical trials.

\section{FASCIA ILIACA BLOCK IN THE EMERGENCY DEPARTMENT-A QUALITY IMPROVEMENT PROJECT, QUEEN ELIZABETH HOSPITAL BIRMINGHAM}

${ }^{1}$ Catherine Browne, ${ }^{2}$ Riad Hosein, ${ }^{3}$ Alistair Jellinek. 'Queen Elizabeth Hospital; ${ }^{2}$ Queen Elizabeth Hospital Birmingham; ${ }^{3}$ University of Birmingham

10.1136/emj-2020-rcemabstracts.39
Aims/Objectives/Background Fractured neck of femur is a common presentation and is associated with high rates of morbidity and mortality. RCEM Best Practice specifies that Fascia Iliaca Block should be available in Emergency Departments as part of the pain management strategy.

\section{Aims}

1. Improve compliance with RCEM guidance for safe administration, documentation and post-procedure monitoring following FIB.

2. Employ QIP methodology to create a FIB protocol.

3. Empower the junior SHO workforce to gain competence in FIB administration through structured teaching.

4. Improve understanding of post-block monitoring in nursing and medical staff.

Methods/Design Data collection identified the number of blocks administered to those presenting with fractured neck of femur in November 2019. Documentation and post-procedure monitoring were evaluated.

Interventions were piloted in January 2020. These were: pre-made block packs, a block checklist sticker incorporating post-procedure monitoring chart and laminated 'quick prompt' guide.

Nurse champions facilitated MDT teaching sessions and junior SHOs were empowered to gain competence in block administration through teaching sessions. 
Retrospective data from January 2020 was compared to November 2019, allowing us to establish the efficacy of changes.

Results/Conclusions October 2019 results demonstrated 59\% of patients received a FIB, this increased to $78 \%$ in January 2020. Pre-intervention, $45 \%$ of patients had the correct dose of local anaesthetic. This increased to $79 \%$ post-intervention. Initially, documentation was correct in just $5 \%$ of cases, improving to $59 \%$ after re-auditing.

Feedback from teaching sessions was positive with nursing staff better understanding the need for post-procedure monitoring. SHOs gained increased confidence delivering FIBs, freeing up senior doctors for other tasks.

The new protocol has improved the administration of FIBs with better post-procedure care and standardised dosing of local anaesthetic. Interventions are embedded in departmental practice; this will be re-audited in 6 months. Following the transition to e-noting we are developing an electronic template to translate these successes onto the new system.

\section{THE MAJOR INCIDENT APP- AN IMPROVED WAY TO CONTACT STAFF IN MAJOR INCIDENTS}

${ }^{1}$ Peter Hulme, ${ }^{1}$ Alannah Madden, ${ }^{2}$ Charlie Reynard. ${ }^{1}$ Manchester Royal Infirmary; ${ }^{2}$ University of Manchester

10.1136/emj-2020-rcemabstracts.40
Aims/Objectives/Background Major incidents are thankfully rare events but require appropriate planning and training to ensure the best response for patients and staff. The method of contacting staff in these incidents is key and needs to be quick, reliable and have excellent response times to be effective. The best method of contacting staff has not been ascertained.

The Major Incident App (MIA) is a new way to contact staff in major incidents and sends loud alerts even when phones are on silent mode. This study looks to compare the major incident response times using MIA with the previous way of contacting staff in a major incident- a freely available digital messaging platform (DMP) at Manchester Foundation Trust.

Methods/Design The decision to declare a major incident or standby was determined by the consultant in Emergency Medicine who was in the department at the time of the incident. The receiving consultants would read the message as soon as they saw it and respond appropriately. After the incidents we conducted a retrospective review of digital communication records to ascertain the time taken for a DMP or MIA message declaring an incident to be seen and read by the receiving consultants.

Reading the message within 15 minutes was regarded as an 'excellent' response time and within 60 minutes was regarded as a 'good' response. These time intervals were determined by the expert opinion of five Major Trauma consultant experts at Manchester Foundation Trust.

\begin{tabular}{|c|c|c|c|c|c|c|c|}
\hline Incident Type & Date & Time & Day of the week & $\begin{array}{l}\text { Ave Response } \\
\text { time mins }\end{array}$ & $\begin{array}{l}\text { Median response } \\
\text { times mins }\end{array}$ & $\begin{array}{l}\text { Seen within } 15 \\
\text { mins (\%) }\end{array}$ & Seen within $1 \mathrm{hr}(\%)$ \\
\hline Simulated & $29 / 3 / 17$ & $10: 38$ & Wednesday & 76.6 & 30 & $9 / 25(36 \%)$ & $15 / 25(60 \%)$ \\
\hline Manchester Arena Attack & $22 / 5 / 17$ & $22: 55$ & Monday & 99.3 & 9.5 & $15 / 26(57.6 \%)$ & $20 / 26(76.9 \%)$ \\
\hline Simulated & $16 / 9 / 17$ & $10: 40$ & Saturday & 79.9 & 10 & $18 / 23(78.2 \%)$ & $22 / 23(95.6 \%)$ \\
\hline City centre Fire & $30 / 12 / 17$ & $17: 29$ & Saturday & 30.5 & 8 & $17 / 25(68 \%)$ & $21 / 25(84 \%)$ \\
\hline Simulated & $18 / 9 / 18$ & 09:35 & Tuesday & 17.8 & 4 & $19 / 24(79.1 \%)$ & $22 / 24(91.7 \%)$ \\
\hline Multiple Stabbing & $11 / 10 / 19$ & 12:05 & Friday & 86.5 & 33.5 & $11 / 28(39.2 \%)$ & $16 / 28(57.1 \%)$ \\
\hline Chemical Incident & $11 / 10 / 19$ & $13: 46$ & Friday & 56.5 & 21.5 & $9 / 26(34.6 \%)$ & $20 / 26(76.9 \%)$ \\
\hline Multiple Stabbing & $15 / 10 / 19$ & $16: 46$ & Tuesday & 23.9 & 11 & $15 / 27(55.5 \%)$ & $25 / 27(92.6 \%)$ \\
\hline New Years Eve multiple traumas & $1 / 1 / 20$ & $04: 14$ & Wednesday & 184.3 & 219 & $4 / 26(15.3 \%)$ & $6 / 26(23 \%)$ \\
\hline Overall & & & & & & $117 / 230(50.8 \%)$ & $167 / 230(72.6 \%)$ \\
\hline
\end{tabular}

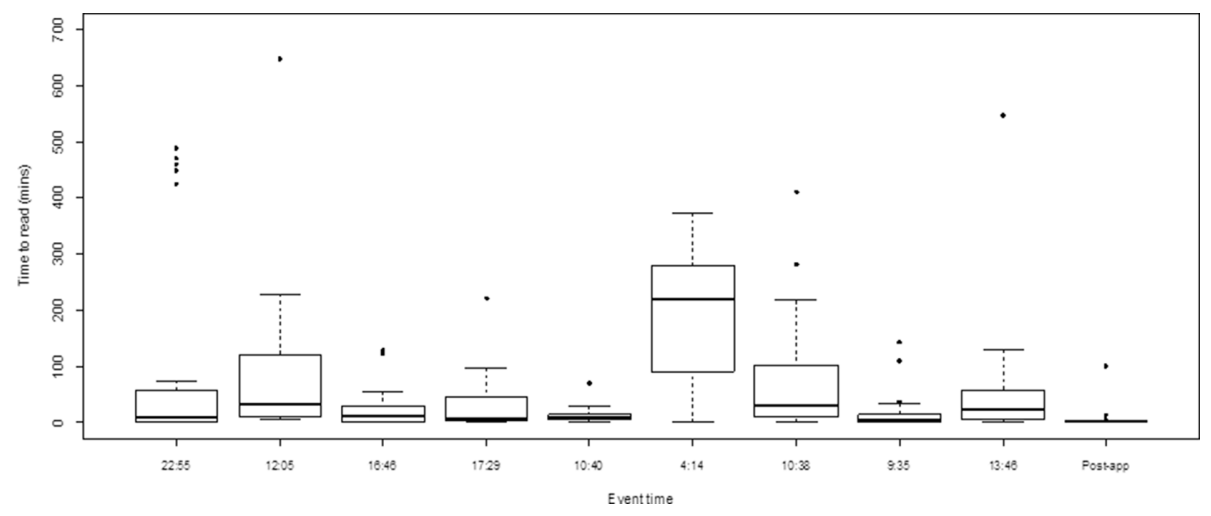

Abstract 134 Figure 1 